\title{
Relationship between CA-125 Levels and Extrauterine Spread in Endometrial Cancer Endometrioid Type at Dr. Soetomo General Hospital Surabaya
}

\author{
Andika Adi Saputra Ahmad, Brahmana Askandar Tjokroprawiro* \\ Division of Gynecologic Oncology, Department of Obstetrics and Gynecology, Dr. Soetomo General Academic Hospital, \\ Medical Faculty - Universitas Airlangga, Surabaya, Indonesia
}

\section{ARTICLE INFO}

Received :

Reviewed :

Accepted :

Keywords:

CA-125, endometrial cancer, extrauterine metastasis

\author{
*Corresponding author: \\ Brahmana Askandar Tjokroprawiro \\ Division of Gynecologic Oncology, \\ Department of Obstetrics and \\ Gynecology, Dr. Soetomo General \\ Academic Hospital, Medical Faculty \\ - Universitas Airlangga, Surabaya, \\ Indonesia \\ brahmanaaskandar@fk.unair.ac.id
}

\begin{abstract}
A BSTRACT
Background: Endometrial cancer is the sixth most common malignancy incidence in women worldwide. The surgical staging in endometrial cancer management such as bilateral salpingooophorectomy and lymphadenectomy is not a routine procedure for all endometrial cancer patients. Therefore, a biomarker to estimate the presence of endometrial cancer metastases to adnexa and lymph nodes is needed. This study aims to find out the level of cancer antigen 125 (CA-125) in endometrial cancer endometrioid type and the relationship between the level of CA-125 and the incidence of adnexal metastases, lymph node metastases, clinical stage, and myometrial invasion.
\end{abstract}

\begin{abstract}
Methods: This observational analytic study used a direct observational design. The in-vitro quantitative examination was performed to measure CA-125 levels using CA-125 Advia Centaur CA-125 Kit. Fischer exact test used to analyzed CA-125 levels with the subject characteristics. All the statistical analyses were performed using SPSS v. 21.0.
\end{abstract}

\begin{abstract}
Results: Of the 30 research subjects with the characteristics of the most age groups of 51-60 years $(60 \%)$, with the most multiparous parity $(73.3 \%)$, grade III $(43.3 \%)$, myometrial invasion > $50 \%$ by $66.7 \%$, early-stage $(63.3 \%)$, the incidence of adnexal metastases $(30 \%)$, and the incidence of lymph node (LN) metastases (10\%), a significant relationship between CA-125 levels and lymph node (LN) metastases was found with a $p=0.041$ and the phi association of 0.016 and between CA-125 levels and adnexal metastases with a $p=0.035$ and the phi association of 0.026 . There was also a significant relationship between CA-125 levels and the clinical stage (advanced stage) with $p=0.004$ and the phi association of 0.002 . There is no significant relationship with a $p=$ 0.702 and a phi association value of -0.098 .
\end{abstract}

Conclusions: CA- 125 can be used as a tumor marker to predict the presence of extrauterine metastases (adnexa \& lymph nodes).

\section{INTRODUCTION}

Endometrial cancer is the sixth most common malignancy incidence in women worldwide. The number of patients with endometrial cancer in developed countries has increased since the mid-20th century [1]. The treatment of endometrial cancer is surgery (complete surgical staging), which includes total hysterectomy, bilateral salpingo-oophorectomy, pelvic \& paraaortic lymphadenectomy, pelvic \& paraaortic lymphadenectomy, and bilateral salpingo-oophorectomy
[2]. This treatment is still controversial in patients with early-stage endometrial cancer and younger patients because it can cause several complications; thus, the Federation of International Gynaecologists and Obstetricians (FIGO) recommends that complete surgical staging be performed only on patients with identified prognostic factors [3]. A preoperative indicator is needed to identify the prognostic factor so that individualistic treatments can be undergone. Now, several predictors can be used in predicting metastases in endometrial 
cancer, including myometrial invasion, tumor size, tumor grading, and tumor histology. These predictors are obtained intraoperatively (frozen section) or preoperatively with MRI [4]. The problem is not all hospitals have this examination. Currently, there is a study on the benefits of CA-125 as a predictor of the presence of endometrial cancer metastases to the adnexa and lymph nodes [5].

This study evaluates the role of CA-125 in predicting the presence of metastases to adnexa and lymph nodes to create more individualistic preoperative preparation and treatment plans in patients with endometrial cancer, neither overtreatment nor undertreatment. CA-125 is a tumor marker that has high sensitivity in predicting the spread of endometrial cancer; however, the specificity of this tumor marker is low due to several conditions such as inflammation, infection, endometriosis cysts, and ovarian cancer [6].

This study is aimed at seeing the relationship between CA-125 levels and the incidence of lymph node metastases, adnexal metastases, and advanced stages of endometrial cancer. This study hypothesizes that if lymph node metastases, adnexal metastases, and the advanced stage of endometrial cancer occur, there will be an increase in CA-125 levels. Therefore, by examining the preoperative CA-125 levels, we can predict and prepare the treatments.

\section{METHODS}

The research design is an observational study in the form of a cross-sectional observational design with a total sample of 30 subjects taken from August 2019 until January 2020. The samples consisting of 30 endometrial cancer patients who had undergone surgical staging did not get any chemoradiotherapy before without any history of endometriosis and adenomyosis primary ovarian cancer. The primary data were collected using direct interviews and examinations from the subject (age, parity, grade, myometrial invasion, stadium, menopausal status, lymph node metastasis, and adnexal metastasis). Serum was taken from venous blood samples before patients underwent surgical staging (operation) for further quantitative in-vitro examination for the measurement of CA-125 by using Kit CA-125 Advia Centaur.

In this study, CA-125 was evaluated to be related to extrauterine endometrial cancer invasion, where the researchers have a different cut-off for CA-125, but most researchers use a cut-off of more than $35 \mathrm{U} / \mathrm{ml}$. The statistical analysis from subject characteristics is presented descriptively; Then, we analyzed CA-125 levels with the incidence of adnexal metastases, lymph node metastases, and advanced stages using the Fischer exact test and used Phi correlation test to determine whether there was a strong correlation between an increase in CA-125 levels more than $35 \mathrm{U} / \mathrm{ml}$ with the incidence of adnexal metastases, lymph node metastases, and advanced stages. With $p<0.05$ and $(+)$ phi correlation means significant. All the statistical analyses were performed using SPSS ver. 21.0.

\section{RESULTS}

Based on the tabulation table, there is a significant relationship between CA-125 levels and lymph node (LN) metastases with a $p=0.041$, and a phi association value of 0.438 . There is a significant relationship between CA-125 levels and the incidence of adnexal metastases with a $p=0.042$ and a phi association value of 0.408 . There is a significant relationship between CA- 125 levels and the clinical stage of endometrial cancer with $p=$ 0.004 and a phi association value of 0.569 . For myometrial invasion, there is no significant relationship with a $p=0.702$ and a phi association value of -0.098 .

\section{DISCUSSION}

Many researchers have been trying to create algorithms that can predict the presence of $\mathrm{LN}$ and adnexal metastases, but it has not yielded satisfactory results. No tumor marker has ever been used as a preoperative marker to predict this. Several studies abroad show that the use of CA-125 as a preoperative marker has satisfactory results and indicates an association between the increase in this marker and the incidence of extrauterine metastases so that bilateral salpingo-oophorectomy and lymphadenectomy are only performed on those with elevated CA-125 levels $[7,8]$. From the results of our study, CA- 125 levels examined preoperatively in each sample of endometrial cancer patients were then correlated with LN metastases, adnexal metastases, and the clinical stage of endometrial cancer. In our study, we set a limit for levels of ca-125 more than $35 \mathrm{U} / \mathrm{ml}$, which is the same limit used by Espino-Strebel and Luna [3], Jiang et al. [8], and Sebastianelli et al. [9]. From these results, we found a significant relationship between increased levels of CA125 and the incidence of lymph node metastases, adnexal metastases, and the advanced stage of endometrial cancer. For metastasis to the lymph node and adnexal, significant values were obtained with $\mathrm{p}=$ 0.041 and 0.042 subsequently and also significant phi association values of 0.0438 and 0.0408 subsequently.

The role of CA-125 in the incidence of metastasis in both lymph nodes and adnexal is through this mechanism due to the bond between Mucin 16 (MUC16)/cancer antigen 125 with E-cadherin and the catenin complex, which causes increased motility, migration, and invasion of tumor cells. In addition, MUC16/CA-125 increases epidermal growth factor receptor (EGFR), which results in increased effectors of Akt and ERK1/2 and increased MMP-2 and MMP-9 expressions. Activation of the MAPK- 
ERK pathway has been shown to increase MMP-9 regulation and enhance cell migration. Through the non-EGFR pathway, CA-125 triggers an increase in $\mathrm{N}$-cadherin, $\mathrm{B}$-catenin, and vimentin and a decrease in E-cadherin and cytokeratin [10].

The occurrence of lymph node metastases and adnexal metastases is closely related to the spread of extrauterine tumors, which is closely related to the occurrence of cell adhesion damage, which we know that the occurrence of adhesions between cells is played by E-cadherin. E-cadherin plays a close role in forming the adherent junction together with catenin ( $\beta$ \& $\alpha$-catenin). E-cadherin is a single transmembrane protein consisting of a complex of 5 calcium ions, which will work with catenin ( $\beta \& \alpha$-catenin) so that it binds to the cytoskeletal actin complex. The bonding mechanism at an adherent junction can take two ways including, a direct signal from integrins which will form dependent integrins and then stimulate bonding at the adherent junction; and the phosphorylation of one of the proteins that play a role in focal adhesion so that phosphorylation of the SRC protein will activate MLCK (myosin light chain kinase), which, after the phosphorylation of MLCK, will induce actin-myosin contractility; besides, actin-myosin contractility was also induced by ROCK (Rho-associated chain kinase) [11].

In addition to a decrease in E-cadherin expression, it was accompanied by an increase in $\mathrm{N}$-cadherin, vimentin, B-catenin, and MMP 9 \& MMP 2. The increase in $\mathrm{N}$-cadherin, vimentin, and $\mathrm{B}$-catenin is closely related to the growth of tumor mass, active movement of tumor mass, and the invasion power of the tumor mass which in turn is closely related to the occurrence of extrauterine metastasis. It was also accompanied by the damage to the extracellular matrix played by MMP 9 and MMP 2 . This could explain the role of CA-125 in the spread of extrauterine tumors, both the incidence of lymph node metastases and adnexal metastases [12].

These results are in accordance with the study conducted by Espino-Strebel and Luna [3]. in 2012 obtaining the same results where there was a significant relationship between increased levels of CA-125 and the incidence of lymph node and adnexal metastases. This is also supported by the study conducted by Sebastianelli et al. [9] in 2010 which found the increased levels of CA-125 in those who have lymph node and adnexal metastases.

This study also found a significant relationship between increased levels of CA-125 and the advanced stage of endometrial cancer because if there are lymph node and adnexal metastases, the patient is classified as having the advanced stage of endometrial cancer. This result is in accordance with the study conducted by Unsal et al. [5] in which CA-125 levels over $35 \mathrm{U} /$ $\mathrm{ml}$ had a significant relationship with the prediction of advanced cancer, myometrial invasion, LVSI, uterine serous involvement, cervical invasion, adnexal involvement, positive peritoneal cytology, and omentum metastases. For the myometrial invasion, it has no relationship with CA-125 levels.

\section{CONCLUSIONS}

The preoperative CA-125 levels of more than $35 \mathrm{U} /$ $\mathrm{ml}$ associated with lymph node metastases, adnexal metastases, and the advanced stage of endometrial cancer. The results of this study are consistent with several other studies.

\section{DECLARATIONS}

\section{Competing of Interest}

The authors declare no competing interest in this study.

\section{Ethics Approval}

This study has been approved by the Ethics Committee of the Dr. Soetomo General Hospital Surabaya, with the ethical clearance certificate no. 1426/KEPK/VI/2019 and has been through AE/SAE monitoring and evaluation data entry and the research subject marking by CRU (Clinical Research Unit) No. 070/0304/CRU/IV/2020 with 30 research subjects.

\section{Acknowledgment}

The author would like to thank the entire academic community at Dr. Soetomo General Hospital for allowing conducting this study and the Department of Obstetrics and Gynecology of Faculty of Medicine, UNAIR, Division of Gynecology Oncology that fully support the implementation of this study from the beginning to completion. Not to forget, the author expresses his gratitude to the Department of Obstetrics and Gynecology of the Faculty of Medicine, University of Hasanuddin as the institution of origin.

\section{REFERENCES}

1. American Cancer Society. Cancer Facts \& Figures 2018 [Internet]. Atlanta: American Cancer Society; 2018 [cited 2021 Feb 18]. Available from: https:// www.cancer.org/content/dam/cancer-org/research/ cancer-facts-and-statistics/annual-cancer-facts-andfigures/2018/cancer-facts-and-figures-2018.pdf

2. Bereck JS, Hacker NF. Uterine Cancer. In: Elfrank JM, editor. Bereck \& Hacker's Gynaecologic Oncology. 6th ed. Philadelphia: Walters Kluwer; 2015. p. 390-442.

3. Espino-Strebel EE, Luna JTP. Correlation between preoperative serum CA 125 and surgicopathologic prognostic factors in endometrial cancer. J Clin Oncol. 2012;27(15): 
4. Sadowski EA, Robbins JB, Guite K, et al. Preoperative pelvic MRI and serum cancer antigen-125: Selecting women with grade 1 endometrial cancer for lymphadenectomy. AJR Am J Roentgenol. 2015;205(5):W556-64.

5. Ünsal M, Comert GK, Karalok A, et al. The preoperative serum CA125 can predict the lymph node metastasis in endometrioid-type endometrial cancer. Ginekol Pol. 2018;89(11):599-606.

6. Felder M, Kapur A, Gonzalez-Bosquet J, et al. MUC16 (CA125): tumor biomarker to cancer therapy, a work in progress. Mol Cancer. 2014;129(13):1-15.

7. Fares $\mathrm{R}$, Kehoe $\mathrm{S}$, Shams N. Preoperative prediction of lymph nodal metastases in endometrial carcinoma: is it possible?: A literature review. Int J Gynecol Cancer. 2018;28(2):394-400.
8. Jiang $T$, Huang $L$, Zhang $S$. Preoperative serum CA125: a useful marker for surgical management of endometrial cancer. BMC Cancer. 2015;15:396.

9. Sebastianelli A, Renaud M-C, Grégoire J, et al. Preoperative CA 125 tumour marker in endometrial cancer: Correlation with advanced stage disease. J Obstet Gynaecol Can. 2010;32(9):856-60.

10. Cao ZQ, Wang Z, Leng P. Aberrant N-cadherin expression in cancer. Biomed Pharmacother. 2019;118:109320.

11. Martinez-Rico C, Pincet F, Thiery JP, Dufour S. Integrins stimulate E-cadherin-mediated intercellular adhesion by regulating Src-kinase activation and actomyosin contractility. J Cell Sci. 2010;123:712-22.

12. Canel M, Serrels A, Frame MC, Brunton V. E-cadherin-integrin crosstalk in cancer invasion and metastasis. J Cell Sci. 2013;126:393-401. 